Faculty of Mathematical Sciences

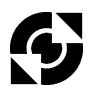

University of Twente

The Netherlands
P.O. Box 217

7500 AE Enschede

The Netherlands

Phone: +31-53-4893400

Fax: +31-53-4893114

Email: memo@math.utwente.nl

www.math.utwente.nl/publications

Memorandum No. 1655

The complexity of the matching-cut

problem for planar graphs

and other graph classes

P.S. Bonsma

OCTOBER, 2002

ISSN 0169-2690 


\title{
The Complexity of the Matching-Cut Problem for Planar Graphs and Other Graph Classes
}

\author{
Paul Bonsma \\ Faculty of Electrical Engineering, Mathematics \& Computer Science \\ Department of Applied Mathematics \\ University of Twente, P.O. Box 217 \\ 7500 AE Enschede, The Netherlands \\ bonsma@math.utwente.nl
}

February 4, 2003

\begin{abstract}
The Matching-Cut problem is the problem to decide whether a graph has an edge cut that is also a matching. Chvátal studied this problem under the name of the Decomposable Graph Recognition problem, and proved the problem to be $\mathcal{N} \mathcal{P}$-complete for graphs with maximum degree 4, and gave a polynomial algorithm for graphs with maximum degree 3. Recently, unaware of Chvátal's result, Patrignani and Pizzonia also proved the $\mathcal{N} \mathcal{P}$-completeness of the problem using a different reduction. They also posed the question whether the Matching-Cut problem is $\mathcal{N} \mathcal{P}$-complete for planar graphs. In this paper an affirmative answer is given. Moreover, it is shown that the problem remains $\mathcal{N} \mathcal{P}$-complete when restricted to planar bipartite graphs, planar graphs with girth 5 and planar graphs with maximum degree 4, making this the strongest result to date. The reduction is from Planar Graph 3-Colorability and differs from the reductions used to prove the earlier results.
\end{abstract}

Keywords: matching, edge cut, decomposable graph, planar graph, computational complexity.

AMS Subject Classification: 05C40,68Q25

\section{Introduction}

A matching in a graph is a subset of the edges such that no two edges in the set share a common end vertex. An edge cut in a graph is a subset of the edges such that the removal of this set results in a disconnected graph. The Matching-Cut problem is the problem to decide whether a given graph has an edge cut that is also a matching. In Section 2 the problem is defined more formally and some basic properties are observed.

Chvátal [1] studied this problem under the name of the Decomposable Graph Recognition problem, and showed that the problem is $\mathcal{N} \mathcal{P}$-complete for graphs with maximum degree 4 
(using the 3-uniform Hypergraph Bicolorability problem), and gave a polynomial algorithm to solve the problem for graphs with maximum degree 3. Later, Patrignani and Pizzonia [5] also proved the $\mathcal{N} \mathcal{P}$-completeness of the problem using a very different reduction (from Not-All-Equal-3-Satisfiability), and gave a linear time algorithm for series-parallel graphs. They also posed the question whether the problem is $\mathcal{N} \mathcal{P}$-complete for the class of planar graphs. In this paper the Matching-Cut problem is shown to be $\mathcal{N} \mathcal{P}$-complete for planar graphs, using a reduction from Planar Graph 3-colorability. This is done in Section 3. By changing the components used in the transformation, in Section 4 the $\mathcal{N} \mathcal{P}$-completeness of the problem is proved for the more restricted classes of planar graphs with maximum degree 4, planar bipartite graphs and planar graphs with girth 5 .

\section{Preliminaries}

For an explanation of the basic graph-theoretical notions used here, we refer to [2]. Cycles and paths can be denoted by ordered lists of vertices and by sets of edges. Both notations will be used.

Let $G=(V, E)$ be a connected graph. A matching is a subset $M \subset E$ such that no two edges in $M$ share an end vertex. An edge cut can be defined as a subset of the edges $M \subseteq E$ such that $(V, E \backslash M)$ is not connected. A matching-cut is a subset $M \subset E$ that is a matching and an edge cut. If $S \subset V$, with $S \neq \emptyset$ and $S \neq V$, then $[S, \bar{S}]$ is the set of edges with exactly one end vertex in $S$ (and one in $\bar{S}$ ). For every $S \subset V$, with $S \neq \emptyset$ and $S \neq V$, there is an edge cut $M$ such that $M=[S, \bar{S}]$. The converse is not true. Still, we will only consider edge cuts that can be written as $[S, \bar{S}]$ for some $S \subset V$, because we are interested in the following decision problem:

\section{Matching-Cut:}

Instance: A graph $G=(V, E)$.

Question: Does $G$ have an edge cut $M \subseteq E$ that is also a matching?

It is easily seen that if a graph has a matching-cut $M$ according to the first definition of an edge cut, then it has a matching-cut of the form $[S, \bar{S}]$.

A graph with a matching-cut is called decomposable, and other graphs indecomposable. We will first consider some small indecomposable graphs, such as triangles $\left(C_{3}\right)$ : if one of the three edges $e$ in a triangle is part of an edge cut, then one of the other edges in this triangle must also be part of the edge cut. Both other edges share an end vertex with $e$, so this edge cut can not be a matching. Therefore we have the following observation:

Observation 1 If edge e is part of a triangle $\left(C_{3}\right)$ of $G$, then there is no matching-cut $[S, \bar{S}]$ in $G$ with $e \in[S, \bar{S}]$.

Similar reasoning yields the next observation:

Observation 2 If $e$ and $f$ are two edges of an induced $C_{4}$ of $G$ that do not share an end vertex and $[S, \bar{S}]$ is a matching-cut in $G$, then $e \in[S, \bar{S}] \Longleftrightarrow f \in[S, \bar{S}]$.

Using this observation we find that $K_{2,3}$ is indecomposable. 
In multigraphs, parallel edges can not be in a matching-cut. Patrignani and Pizzonia [5] observed that for any multigraph $G$, a simple graph $G^{\prime}$ can be constructed such that $G$ has a matching-cut if and only if $G^{\prime}$ has a matching-cut: in the multigraph, replace all sets of parallel edges by two parallel edges and subdivide one of these with one vertex, giving a triangle. Therefore we will use multigraphs, but the results we prove also hold for simple graphs.

For more information on the subject of algorithms and complexity, we refer to [3].

\section{The $\mathcal{N} \mathcal{P}$-completeness of Planar Matching-Cut}

In this section, the $\mathcal{N} \mathcal{P}$-completeness of the following problem is proved:

\section{Planar Matching-Cut:}

Instance: A graph planar $G=(V, E)$.

Question: Does $G$ have an edge cut $[S, \bar{S}]$ that is also a matching?

This $\mathcal{N} \mathcal{P}$-completeness proof is by a polynomial transformation from the following graph coloring problem:

\section{Planar Graph 3-Colorability}

Instance: A planar graph $G=(V, E)$

Question: Does $G$ have a vertex coloring using at most 3 colors?

Theorem 1 Planar Graph 3-Colorability is $\mathcal{N} \mathcal{P}$-complete.

This has been shown in [4]. In order to prove the $\mathcal{N} \mathcal{P}$-completeness of Planar MatchingCut, two intermediate problems will be used which are shown to be $\mathcal{N} \mathcal{P}$-complete. For all problems in this section, membership of the class $\mathcal{N} \mathcal{P}$ is obvious, so only $\mathcal{N} \mathcal{P}$-hardness of the problems is shown. First we show that Graph 3 -Colorability is still $\mathcal{N} \mathcal{P}$-hard when restricted to a smaller set of instances:

\section{Planar Hamiltonian Graph 3-Colorability:}

Instance: A planar graph $G=(V, E)$ with a Hamilton cycle $H \subseteq E$.

Question: Does $G$ have a vertex coloring using at most 3 colors?

Lemma 2 Planar Hamiltonian Graph 3-Colorability is $\mathcal{N} \mathcal{P}$-complete.

Proof: Let $G=(V, E)$ be a planar graph. We use $G$ to construct a planar Hamiltonian graph $G^{\prime}=\left(V^{\prime}, E^{\prime}\right)$ such that $G$ is 3-colorable if and only if $G^{\prime}$ is 3-colorable. Using Theorem 1 , this proves the $\mathcal{N} \mathcal{P}$-completeness.

First observe that a graph $G$ with a vertex $v$ with $d(v)<3$ is 3-colorable if and only if $G-v$ is 3-colorable. To construct $G^{\prime}=\left(V^{\prime}, E^{\prime}\right)$ we will start with an embedding of $G=(V, E)$. First edges will be added to make the graph Hamiltonian. If this introduces crossings, these are replaced by the crossing components that are defined below, restoring planarity. This replacement does not remove vertices, so $V \subset V^{\prime}$. We then proceed by showing that any proper 3-coloring of $G$ can be extended to a proper 3-coloring of $G^{\prime}$ and any proper 3-coloring of $G^{\prime}$ restricted to $V$ is a proper 3-coloring of $G$. 


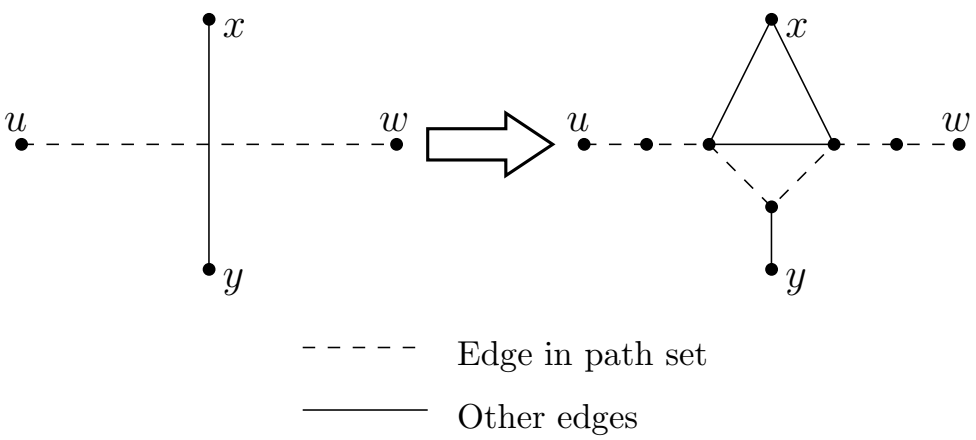

Figure 1: Removing a crossing

For the first step, we first try to find a Hamilton cycle in $G$ with the following simple procedure:

Start at an arbitrary vertex $v_{1}$.

for $i=1, \ldots,|V(G)|-1$ :

if vertex $v_{i}$ has a neighbor $u \notin\left\{v_{1}, \ldots, v_{i-1}\right\}$ then

$$
v_{i+1}:=u
$$

else choose an arbitrary vertex $v_{i+1} \notin\left\{v_{1}, \ldots, v_{i}\right\}$

This procedure constructs a set of vertex disjoint paths $Q_{1}, \ldots, Q_{k}$ containing all vertices of $G$ (some paths may contain only one vertex). If $k>1$, take the paths $Q_{1}$ and $Q_{2}$ and end vertex $u=v_{j}$ of $Q_{1}$ and end vertex $v=v_{j+1}$ of $Q_{2}$. Now add a new vertex $w$ and edges $(u, w)$ and $(v, w)$. Connect the paths using $w$ such that the number of paths $k$ decreases. Repeat this (with new vertices in the role of $w$ ) until $k=1$. If $k=1$, we have a Hamilton path and with a choice of $u=v_{1}$ and $v=v_{n}$ with $n=|V(G)|$, the above procedure gives a Hamilton cycle. So this procedure constructs a graph with a given Hamilton cycle in a linear number of steps. Because $d(w)=2$, in each step the addition of a vertex $w$ has no influence on the 3-colorability of the graph.

Adding the edges $(u, w)$ and $(v, w)$ may not be possible without destroying planarity. Therefore, each addition is done as follows: in the embedding of $G$, the edges of the paths $Q_{1}, \ldots, Q_{k}$ do not divide the plane in more than one region. Therefore an edge $(u, v)$ can be drawn such that it does not cross any edges used in the paths, and crosses each of the other edges at most once. Subdivide $(u, v)$ using $w$, creating $(u, w)$ and $(v, w)$. This subdivision can be done in such a way that on $(v, w)$ there are no crossings. Iteratively remove crossings on $(u, w)$ as follows: if $(u, w)$ crosses an edge $(x, y)$, replace this crossing with the crossing component shown in Figure 1 (without introducing new crossings). This crossing component has the following two properties: in a proper 3-coloring, $u$ and $w$ can have any color assigned to them, independent of the color assigned to the other vertex and the vertices $x$ and $y$. Furthermore, $x$ and $y$ will be colored differently. Note that all new vertices in the crossing component are part of the new path from $u$ to $w$ and that $(x, y)$ was not part of the path set, so we still have a vertex disjoint set of paths containing all vertices. Repeating this 
procedure removes all crossings.

Let $G^{\prime \prime}$ be the graph obtained after adding edges $(u, w)$ and $(v, w)$ and removing all crossings in the described way. Then any proper 3-coloring of $G$ can be extended to a proper 3-coloring of $G^{\prime \prime}$, because the new neighbors of $u$ and $v$ have degree 2. Any proper 3 -coloring of $G^{\prime \prime}$ is a proper 3-coloring of $G$ when restricted to $V$, because a proper 3-coloring of a crossing component assigns different colors to $x$ and $y$.

So if this procedure of adding edges and removing crossings is repeated until a planar graph $G^{\prime}$ with a Hamilton cycle is constructed, $G^{\prime}$ is 3-colorable if and only if $G$ is 3-colorable. This completes the $\mathcal{N} \mathcal{P}$-completeness proof.

Before we can use Graph 3-Colorability to prove the $\mathcal{N} \mathcal{P}$-completeness of Planar MatchingCut, we transform the problem into an artificial, but more suitable vertex coloring problem in which every vertex is incident with only one vertex that has to be colored with a different color, and at most two that have to be colored with the same color:

\section{Segment 3-Colorability}

Instance: A set of vertices $V$ and three disjoint edge sets $A, B$ and $C$ such that $G=$ $(V, A \cup B \cup C)$ is a 3-regular planar multigraph with Hamilton cycle $A \cup B$.

Question: Can we find a color function $f: V \rightarrow\{1,2,3\}$ such that if $u v \in A$ then $f(u)=f(v)$ and if $u v \in C$ then $f(u) \neq f(v)$ ?

An instance of Segment 3-Colorability will be denoted by the multigraph $G=(V, A \cup B \cup C)$. Note that this instance gives a multigraph, so if for instance $B$ and $C$ both contain an edge between vertices $u$ and $v$, in $G$ these are considered to be different edges. Edges in the sets $A, B$ and $C$ will be called respectively $A$-edges, $B$-edges and $C$-edges. If $G=(V, A \cup B \cup C)$ is an instance of Segment 3-Colorability, the components of the graph $(V, A)$ are called segments. All vertices of one segment receive the same color in a solution of this problem, and therefore the problem is called segment coloring. Note that $C$ is a perfect matching in $G$.

Using lemma 2, we can prove the $\mathcal{N} \mathcal{P}$-completeness of Segment 3-Colorability:

Lemma 3 Segment 3-Colorability is $\mathcal{N} \mathcal{P}$-complete.

Proof: We will construct an instance $G^{\prime}$ of Segment 3-Colorability from an instance $G$ of Planar Hamiltonian Graph 3-Colorability such that:

- the vertices of $G$ correspond to the segments of $G^{\prime}$,

- the edges of $G$ correspond to the edge set $C$ of $G^{\prime}: G^{\prime}$ has a $C$-edge between vertices of segments $x$ and $y$ if and only if there is an edge between the vertices of $G$ corresponding to $x$ and $y$, and

- the vertex ordering given by the Hamilton cycle $H$ in $G$ is the same as the segment ordering given by the Hamilton cycle $A \cup B$ in $G^{\prime}$.

Using the first two properties above, it is easy to see that Segment 3-Colorability on $G^{\prime}$ is equivalent with Planar Hamiltonian Graph 3-Colorability on G. An example of this transformation is shown in Figure 2.

Suppose an embedding of $G$ in the plane is known. The transformation uses the following steps, which are shown in Figure 3: 


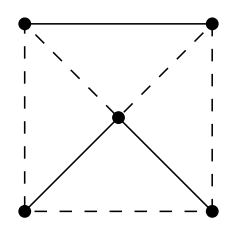

Edges in $G$ :

- - - - Edge in Hamilton cycle Other edges

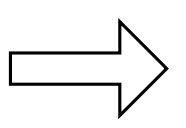

Edges in $G^{\prime}$ :

Edge in $A$

Edge in $B$

Edge in $C$

Figure 2: An example of the transformation in the proof of Lemma 3
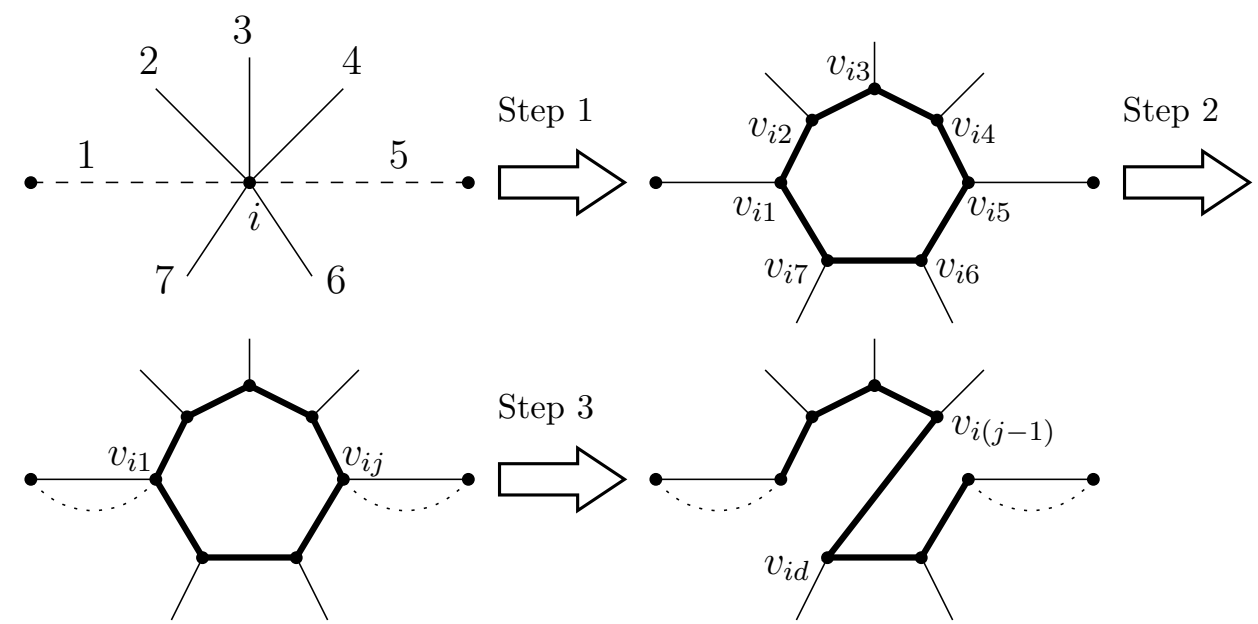

Edges in $G$ :

Edges in $G^{\prime}$ :

- - - Edge in Hamilton cycle

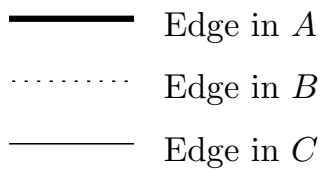

Figure 3: The transformation steps in the proof of Lemma 3 
1. For each vertex $i$ in $G$, we can use the embedding to find a clockwise ordering of the incident edges (such that edge $j$ and edge $j+1$ are on the boundary of the same face). For every incident edge $j$, subdivide it using vertex $v_{i j}$. Add edges $\left(v_{i j}, v_{i(j+1)}\right)(j=i, \ldots, d(i)-1)$ and $\left(v_{i d}, v_{i 1}\right)$, where $d=d(i)$. Delete vertex $i$. The edges corresponding to the edges of $G$ form the edge set $C$, and the new edges form the edge set $A$.

2. Double every edge in $C$ corresponding to an edge in $G$ that is used in the Hamilton cycle $H$. The new edges form the edge set $B$.

3. For every $i$, suppose w.l.o.g. that $v_{i 1}$ and $v_{i j}$ are the two vertices on the cycle that are incident with edges in $B$. Let $d=d_{G}(i)$. Now delete the edges $\left(v_{i(j-1)}, v_{i j}\right)$ and $\left(v_{i 1}, v_{i d}\right)$ and add an edge $\left(v_{i(j-1)}, v_{i d}\right)$. The new edge belongs to the edge set $A$. Now the edges of $A$ form a Hamilton path from $v_{i 1}$ to $v_{i j}$ in the subgraph induced by $v_{i 1}, \ldots, v_{i d}$.

This transformation yields a 3-regular graph $G^{\prime}$. All steps used in the transformation preserve planarity so $G^{\prime}$ is planar. (These steps are: subdivision of edges with vertices, doubling edges, adding edges between vertices that are on the boundary of the same face and deleting vertices and edges.)

For every $i$, vertices $v_{i j}\left(j=1, \ldots, d_{G}(i)\right)$ induce a component of $\left(V\left(G^{\prime}\right), A\right)$ and therefore receive the same color in a feasible solution of Segment 3-Colorability. If (and only if) an edge $i j$ is present in $G$, a $C$-edge $v_{i x} v_{j y}$ is present in $G^{\prime}$ for some $x$ and $y$, so segments $i$ and $j$ receive different colors in a feasible Segment 3-Colorability solution for $G^{\prime}$. We conclude that a vertex 3-coloring of $G$ can be transformed into a segment 3-coloring of $G^{\prime}$ and vice versa. So Segment 3 -Colorability is $\mathcal{N} \mathcal{P}$-complete.

Now, using Lemma 3 we can prove the $\mathcal{N} \mathcal{P}$-completeness of Planar Matching-Cut.

Theorem 4 Planar Matching-Cut is $\mathcal{N} \mathcal{P}$-complete

Proof: For this proof, we transform an instance $G=(V, A \cup B \cup C)$ of Segment 3-Colorability into an instance $G^{\prime}$ of Planar Matching-Cut. For every vertex in $V$ we will introduce a vertex component in $G^{\prime}$. (Throughout this proof, we do not use the graph theoretical meaning of the word component.) If two vertices in $V$ are joined by an $A$-edge, the corresponding vertex components will be connected using a vertex connection component. If two vertices in $V$ are joined by a $B$-edge, the corresponding vertex components will be connected using a segment connection component. In order to properly label the components that will be introduced, first label the vertices of $G$ with labels $1, \ldots, k_{1}$. Label the edges in $A$ with labels $k_{1}+1, \ldots, k_{2}$. Label the edges in $B$ with $k_{2}+1, \ldots, k_{3}$. Note that for $C$-edges no components will be introduced.

Orient all edges of the Hamilton cycle $A \cup B$ anticlockwise with regard to the embedding of $G$. Orient the edges in $C$ arbitrarily. Since the edge set $A \cup B$ gives a Hamilton cycle, in the embedding of $G$, this Hamilton cycle divides the plane into two regions. So we can divide the edges of $C$ into two categories using this Hamilton cycle: inside edges and outside edges. Because every vertex $i$ in $G\left(i=1, \ldots, k_{1}\right)$ is incident with exactly one edge $e \in C$, we can use this to define four variants of vertex components: 


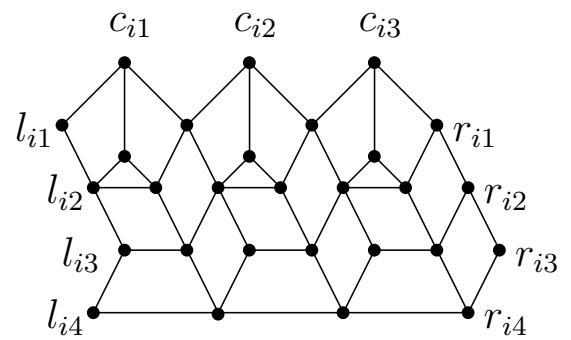

(a) $i$ incident with tail of inside edge

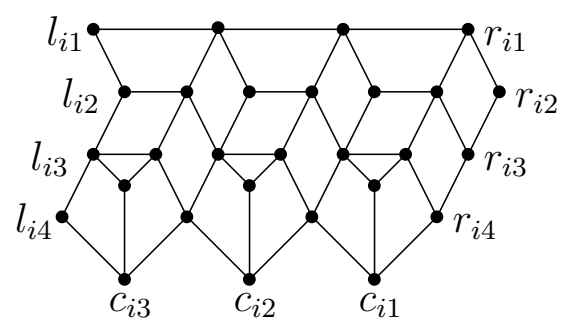

(c) $i$ incident with tail of outside edge

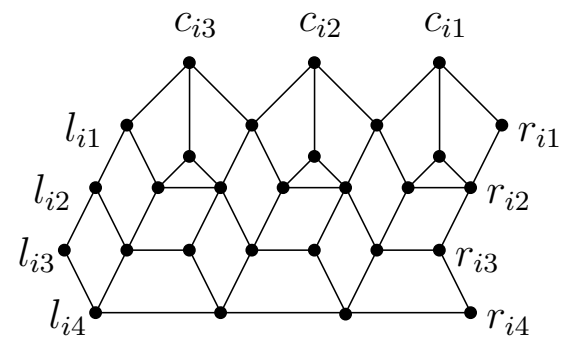

(b) $i$ incident with head of inside edge

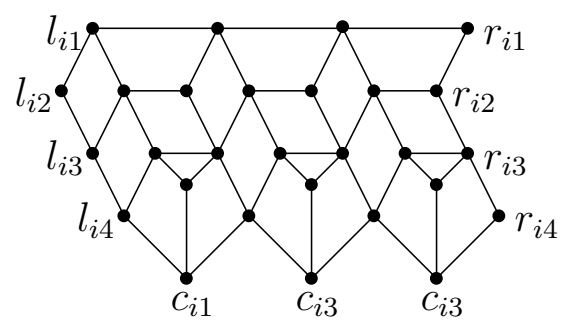

(d) $i$ incident with head of outside edge

Figure 4: Four variants of vertex components for $G^{\prime}$

1. If $e$ is an inside edge and $i$ is incident with the tail of $e$, introduce a vertex component as shown in Figure 4a.

2. If $e$ is an inside edge and $i$ is incident with the head of $e$, introduce a vertex component as shown in Figure $4 \mathrm{~b}$.

3. If $e$ is an outside edge and $i$ is incident with the tail of $e$, introduce a vertex component as shown in Figure 4c.

4. If $e$ is an outside edge and $i$ is incident with the head of $e$, introduce a vertex component as shown in Figure 4 d.

There are at least three possible matching-cuts through these components, which are shown in Figure 5. We use the two observations from Section 2 to show that these are the only possible matching-cuts: if one edge of a matching-cut through a vertex component is given, the other edges in the matching-cut can be derived, because all edges are on a (facial) 4-cycle. Observe that if one of the edges that is not part of the three given matching-cuts is in a matching-cut, this will imply that an edge of one of the triangles is part of the matching-cut, a contradiction. Therefore these are the only three possible matching-cuts through a vertex component. Note that no two of these matching-cuts can occur simultaneously.

If edge $l_{i j} l_{i(j+1)}$ is in the matching-cut, we will say that this vertex component is cut by cut $j(j=1,2,3)$. Below, this will correspond to a coloring of vertex $i$ in $G$ with color $j$. Observe that in this case, $r_{i j} r_{i(j+1)}$ is also in the matching-cut, and vertex $c_{i j}$ is incident 

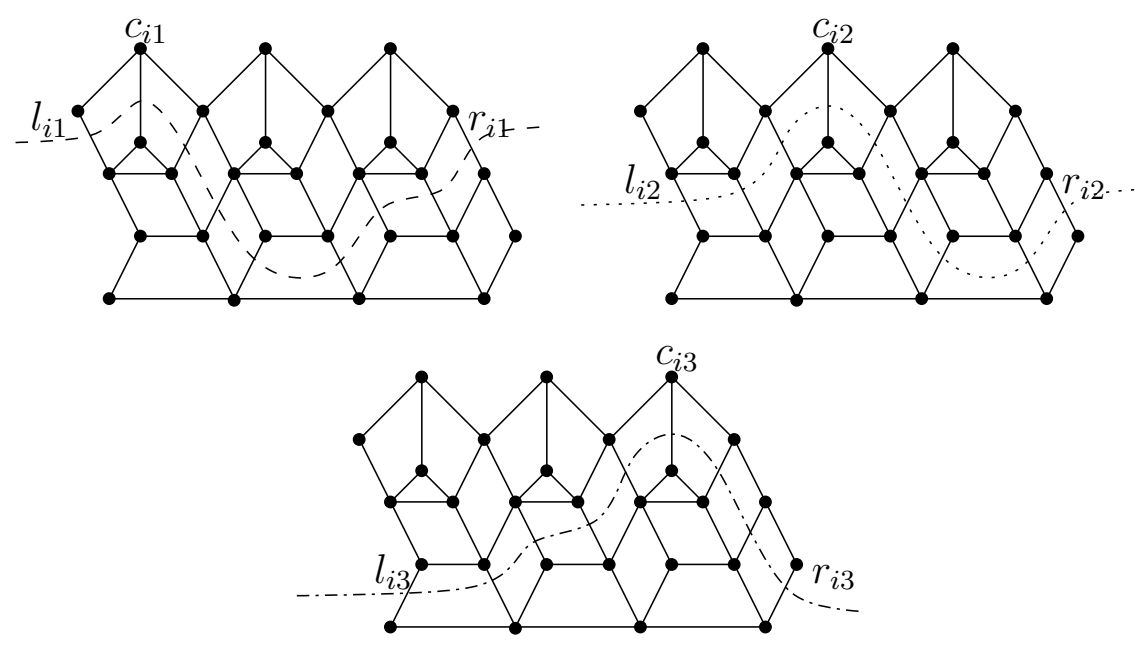

Figure 5: The three possible matching-cuts through a vertex component

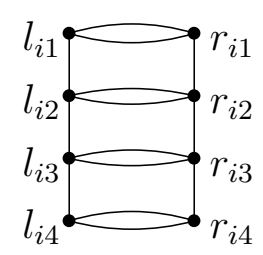

Figure 6: A vertex connection component

with one of the edges in the matching-cut, whereas $c_{i k}$ for $k \neq j$ is not.

For each edge $i$ in $A\left(i=k_{1}+1, \ldots, k_{2}\right)$, we introduce a vertex connection component as shown in Figure 6.

It is easy to see that there are exactly three possible matching-cuts through these components. Observe that if edge $l_{i j} l_{i(j+1)}$ is in the matching-cut, then edge $r_{i j} r_{i(j+1)}$ also is.

If in $G$, there is an $A$-edge $k$ from $i$ to $j$, the two vertex components $i$ and $j$ will be connected by vertex connection component $k$ as follows: identify $r_{i l}$ with $l_{k l}$ for $l=1,2,3,4$. This new vertex is called $r_{i l}$. Now, for $l=1,2,3$ there are two edges from $r_{i l}$ to $r_{i(l+1)}$. Delete one of these. Next, identify $r_{k l}$ with $l_{j l}$ for $l=1,2,3,4$ and call the new vertices $l_{j l}$. Also delete one of all the double edges introduced here. Such a vertex connection has the following property:

Property 1 If two vertex components $i$ and $j$ are connected by a vertex connection component, then for any matching-cut: $i$ is cut by cut $l$ if and only if $j$ is cut by cut $l(l=1,2,3)$. 

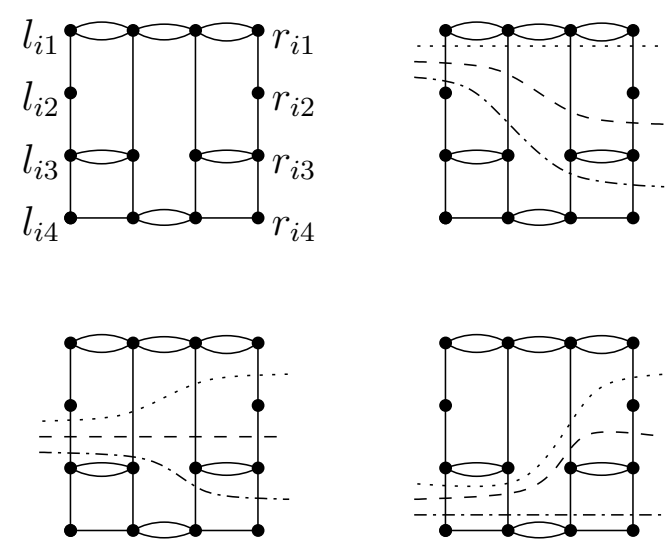

Figure 7: A segment connection component and the nine possible matching-cuts

Clearly, to achieve this property, vertex components can also be connected without using a vertex connection component, but for the alternative constructions in the next section, vertex connection components are useful.

For each edge $i$ in $B\left(i=k_{2}+1, \ldots, k_{3}\right)$, we introduce a segment connection component as shown in Figure 7.

Because the double edges cannot be in a matching-cut, there are nine possible matchingcuts through these components, all of which cut exactly one edge $l_{i j} l_{i(j+1)}$ and one edge $r_{i k} r_{i(k+1)}$. There is one cut for every combination of $j$ and $k(j=1,2,3, k=1,2,3)$.

If in $G$ there is a $B$-edge from $i$ to $j$, we can connect the vertex components $i$ and $j$ using a segment connection component in the same way as described above for vertex connection components. This segment connection has the following property:

Property 2 If two vertex components $i$ and $j$ are connected by a segment connection component, then for any matching-cut: $i$ is cut by this matching-cut if and only if $j$ is cut by this matching-cut. Any combination of cuts through $i$ and $j$ is possible.

The $C$-edges of $G$ determine the last type of connection between vertex components: if $i j \in C$, identify $c_{i l}$ with $c_{j l}(l=1,2,3)$. This connection is called an edge connection. We know that if vertex component $i$ is cut by cut $l$, then $c_{i l}$ is incident with an edge of this matching-cut. So because a matching-cut is a matching, this connection has the following property:

Property 3 If two vertex components $i$ and $j$ are connected by an edge connection, then for any matching-cut and $l=1,2,3$ : it is not possible that $i$ is cut by cut $l$ and $j$ is cut by cut $l$.

Let $G^{\prime}$ be the graph that is constructed by introducing vertex components for every vertex in $G$ and connecting them with vertex connection components, segment connection components 
and edge connections for every edge in respectively $A, B$ and $C$ as described above. Using the observed properties of these connections, $G^{\prime}$ has the following properties:

- Because $A \cup B$ gives a Hamilton cycle in $G$, and the fact that the different components have no matching-cuts other than the indicated cuts, we use Property 1 and Property 2 to conclude that if $G^{\prime}$ has a matching-cut, this matching-cut cuts every vertex component.

- Property 1 show that if $G^{\prime}$ has a matching-cut, all vertex components that correspond to vertices in the same segment of $G$ are cut by the same cut $l$.

- By Property 3 , in any matching-cut, two vertex components that correspond to vertices in segments that are joined by a $C$-edge must be cut by different cuts.

- Property 2 show that segment connection components do not impose additional constraints on the possible combinations of cuts through vertex components.

Now it is easy to see how any matching-cut in $G^{\prime}$ corresponds to a proper segment 3-coloring of $G$ and vice versa.

The only thing left to prove is that $G^{\prime}$ is indeed an instance for Planar Matching-Cut. Therefore, we show that $G^{\prime}$ is planar.

$|V|$ vertex components, $|A|$ vertex connection components and $\mid B$ segment connection components can be drawn in the plane, with inner faces as shown in the figures, and all sharing outer face $F$. Because $A \cup B$ is a Hamilton cycle in $G$, making the vertex connections and segment connections does not destroy the planarity, but does split face $F$ into two faces: $F_{1}$ and $F_{2}$. This is because every vertex $l_{i l}$ and $r_{i l}$ is used exactly once in these connections. Assume w.l.o.g. that every vertex $l_{i 1}$ and $r_{i 1}$ is on the boundary of $F_{1}$, and every vertex $l_{i 4}$ and $r_{i 4}$ is on the boundary of $F_{2}$.

If a $C$-edge $i j$ is present, then vertex components $i$ and $j$ are either of type (a) and (b) as shown in Figure 4, or of type (c) and (d) as shown in this figure. Therefore either $c_{i k}$ and $c_{j k}(k=1,2,3)$ are all on the boundary of $F_{1}$, or they are all on the boundary of $F_{2}$. Also, edges of $C$ do not cross in the embedding of $G$. Because of this, all such vertex pairs can be identified without destroying the planarity. This gives an embedding of $G^{\prime}$.

Since the given transformation is polynomial, and Planar Matching-Cut is within $\mathcal{N P}$, Planar Matching-Cut is $\mathcal{N} \mathcal{P}$-complete.

\section{The $\mathcal{N} \mathcal{P}$-completeness of Matching-Cut for Several Other Graph Classes}

\subsection{Planar graphs with maximum degree 4}

As Chvátal proved the Matching-Cut problem to be $\mathcal{N} \mathcal{P}$-complete for graphs with maximum degree 4 , the question arises whether the problem is also $\mathcal{N} \mathcal{P}$-complete for planar graphs with maximum degree 4 . To prove that this is indeed the case, we outline how the construction used in the proof of Theorem 4 should be altered such that the resulting graph $G^{\prime}$ is a planar graph with maximum degree 4 , and still has the properties needed in the proof. 


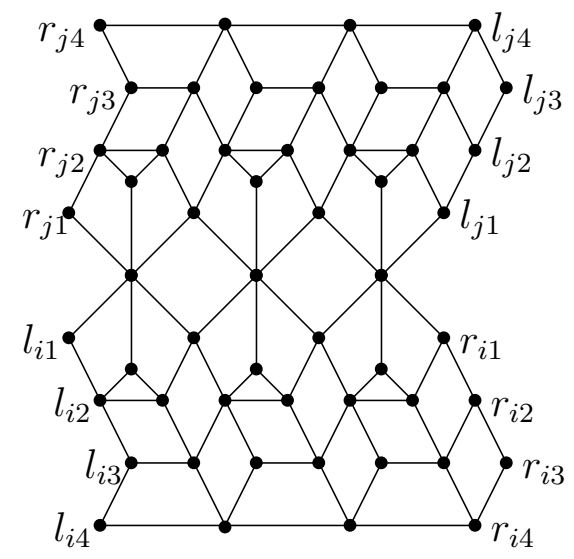

Figure 8: Two vertex components form an edge component

For this alteration, in the graph $G^{\prime}$ from the proof of Theorem 4, we will replace all vertex connection components by new vertex connection components, and do the same for all segment connection components. The vertex components will be replaced pairwise: in $G^{\prime}$, every vertex component occurs in a pair as shown in Figure 8 (this pair corresponds to an inside $C$-edge in the Segment 3-Colorability instance. For outside edges the labeling is different, but this is not important). Call this subgraph, composed of vertex components $i$ and $j$, edge component $i j$.

Replace each edge component $i j$ in $G^{\prime}$ by the new edge component $i j$ shown in Figure 9(a). This component is obviously planar and has maximum degree 4 . Furthermore, the vertices $l_{i k}, r_{i k}, l_{j k}$ and $r_{j k}(k=1,2,3,4)$ have smaller degree such that further connections are possible. With a little effort it can be checked (using the observations in Section 2)that the possible combinations of matching-cuts through this new edge component are similar to the possible combinations of matching-cuts through the edge component in Figure 8. There is one difference: the matching-cuts are 'reversed', so for instance the matching-cut that contains the edge $l_{i 1} l_{i 2}$ also contains the edge $r_{i 3} r_{i 4}$ and the matching-cut that contains the edge $l_{i 3} l_{i 4}$ also contains the edge $r_{i 1} r_{i 2}$. This is easily remedied by replacing each edge component in $G^{\prime}$ be two of the edge components in Figure 9(a). Connect the vertices $r_{i k}$ of the first copy to the vertices $l_{i k}$ of the second copy, and the vertices $l_{j k}$ of the first copy to the vertices $r_{j k}$ of the second copy $(k=1,2,3,4)$, and remove double edges.

Replace each segment connection component (Figure 7) by the component shown in Figure 9(b). This component is again planar, has maximum degree 4 and has a set of matching-cuts equivalent to the nine matching-cuts of the original segment connection component.

For the vertex connection components we use the component shown in Figure 9(c).

If we replace all subgraphs of $G^{\prime}$ exactly as described, then next to edge components corresponding to outside edges, vertices of degree 5 will occur. This problem can easily be solved by inserting extra vertex connection components (with a double edge between $l_{l 4}$ and $r_{l 4}$ instead of between $l_{l 1}$ and $r_{l 1}$ ) and/or deleting vertex connection components. 


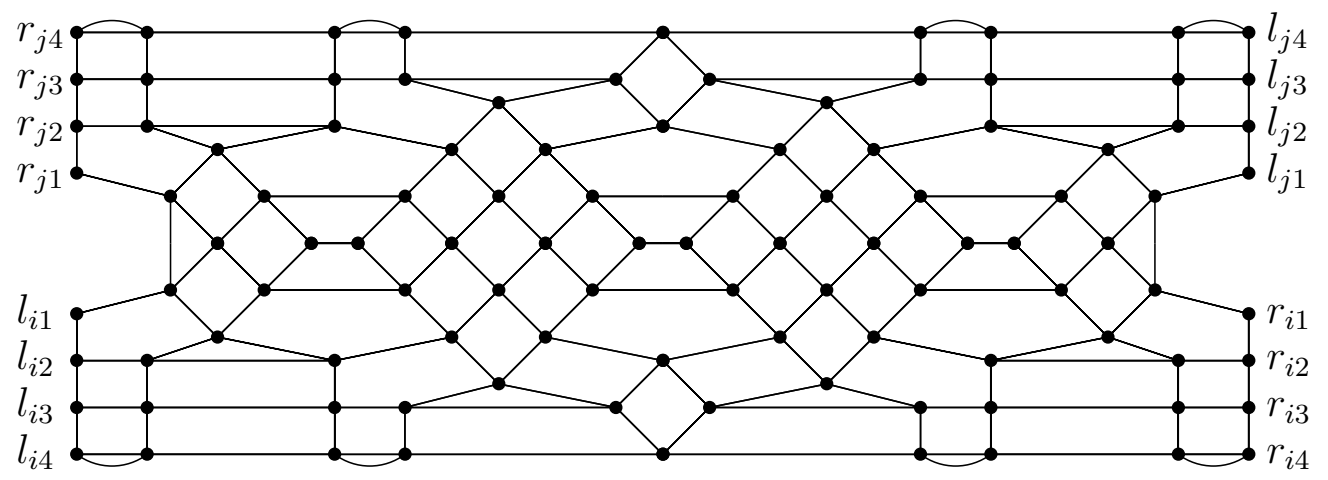

(a) Edge component $i j$

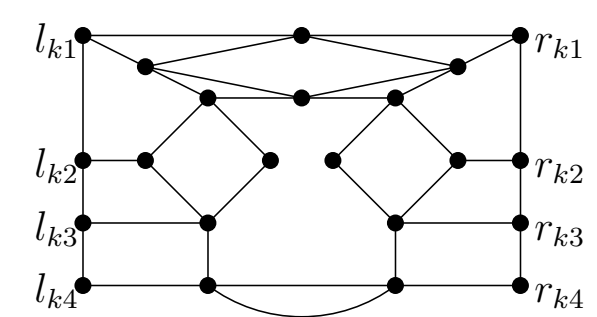

(b) Segment connection component $k$

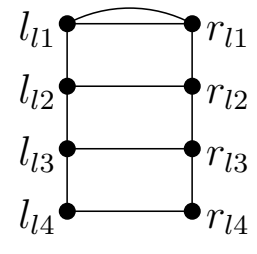

(c) Vertex connection component $l$

Figure 9: Components with maximum degree 4 


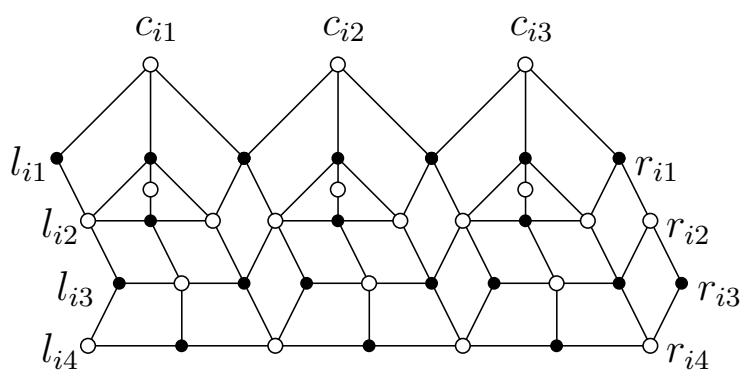

(a) Vertex component $i$

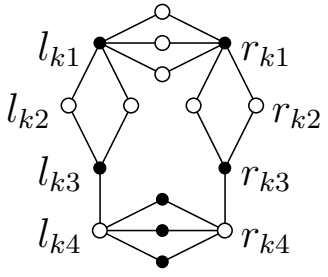

(b) Segment connection component $k$

Figure 10: Bipartite components

This shows how the construction can be altered such that the resulting graph has maximum degree 4 and still has all the necessary properties. Therefore, the Matching-Cut problem is $\mathcal{N} \mathcal{P}$-complete for the class of planar graphs with maximum degree 4 .

\subsection{Planar Bipartite Graphs}

After we observe that the indecomposable graphs that we have seen all contain small cycles (triangles), the question arises whether the Matching-Cut problem is still $\mathcal{N} \mathcal{P}$-complete for graphs with large girth (the girth of a graph is the length of a shortest cycle). First consider simple graphs without triangles or, for a stronger result, bipartite simple graphs. $K_{2,3}$ is the smallest indecomposable graph without triangles. This graph can be used to construct components that can be used to prove the $\mathcal{N} \mathcal{P}$-completeness of the Matching-Cut problem for planar bipartite graphs.

For instance, replace in the graph $G^{\prime}$ used in the proof of Theorem 4 each vertex component by the bipartite vertex component $i$ shown in Figure 10(a) and replace each segment connection component in $G^{\prime}$ by the segment connection component $k$ shown in Figure 10(b). Recall that in the proof it was observed that vertex connection components are not needed in $G^{\prime}$. Further details are left to the reader.

\subsection{Planar Graphs with Girth Five}

Before we can prove that the Matching-Cut problem is $\mathcal{N} \mathcal{P}$-complete for planar graphs with girth 5, we must find such a graph that is indecomposable. First we define the operation of inserting a graph in a face of another graph. Let $G$ be a plane graph with $C_{1}$ a cycle of length $d$ corresponding to an inner face. Let $H$ be a plane graph such that $C_{2}$ is the cycle of length $d$ corresponding to the outer face. First, $H$ can be drawn in the inner face of $G$ corresponding to $C_{1}$. All vertices of $C_{1}$ can be identified with vertices of $C_{2}$ such that the resulting graph is still planar (this can be done in $d$ ways). Delete one of all double edges produced this way in order to obtain a simple graph. Call this operation inserting $H$ into $C_{1}$ of $G$. If $G$ and $H$ have girth at least 5 , and for any two vertices $u$ and $v$ on the cycle 


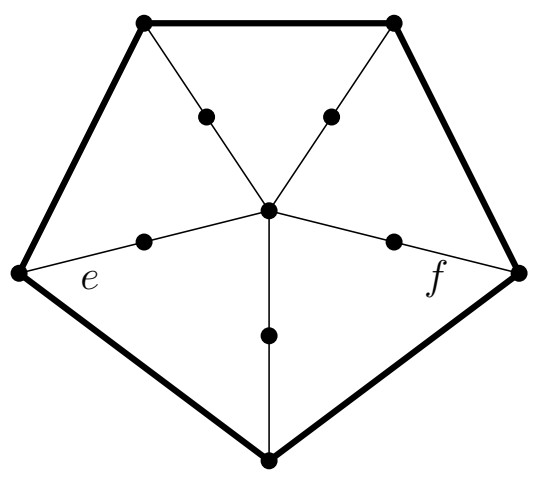

(a)

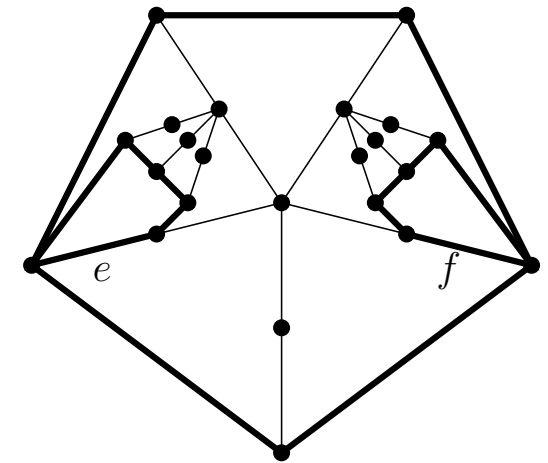

(b)

Figure 11: Making an indecomposable graph with girth 5

$C_{1}\left(C_{2}\right)$, the distance from $u$ to $v$ in $G(H)$ is either equal to the distance from $u$ to $v$ on the cycle $C_{1}\left(C_{2}\right)$ or is at least 3 , then the girth of the resulting graph is again at least 5 .

In Figure 11(a), a planar graph with girth 5 is shown that has the property that none of the thick edges can be part of a matching-cut. However, this graph does have six different matching-cuts, all of which contain at least one of the edges $e$ and $f$. In Figure 11(b), the same graph is shown, but to make sure that edges $e$ and $f$ can not be part of a matching-cut, two additional copies of the graph in Figure 11(a) are inserted in two of the faces (a different embedding of the graph is used, in which one of the inner faces becomes the outer face). Now none of the thick edges can be part of a matching-cut, while girth 5 and planarity is maintained. Considering these thick edges, it can be checked that the graph in Figure 11(b) is indecomposable. In a similar way, for any $d \geq 5$ embeddings of indecomposable planar graphs with girth 5 can be constructed such that the outer face has degree $d$. Call such a graph an indecomposable perimeter-d graph.

Now we can outline how to change the proof of Theorem 4 such that the resulting graph is a planar graph with girth 5, which proves the $\mathcal{N} \mathcal{P}$-completeness of the Matching-Cut problem for planar graphs with girth 5. Consider the components in Figure 12. Replace edge components and segment connection components in the graph $G^{\prime}$ from the proof of Theorem 4 by these new components. Recall that vertex connection components do not have to be used. Now, edges on the boundary of the shaded faces can be subdivided until all 2-cycles, 3-cycles and 4-cycles are removed (observe that in the graph obtained from $G^{\prime}$, all these small cycles contain such an edge). To ensure that none of the edges around the shaded faces can be part of a matching-cut, insert in every shaded face of degree $d$ an indecomposable perimeter- $d$ graph. This yields a planar graph of girth 5 that has the desired matching-cut properties. 


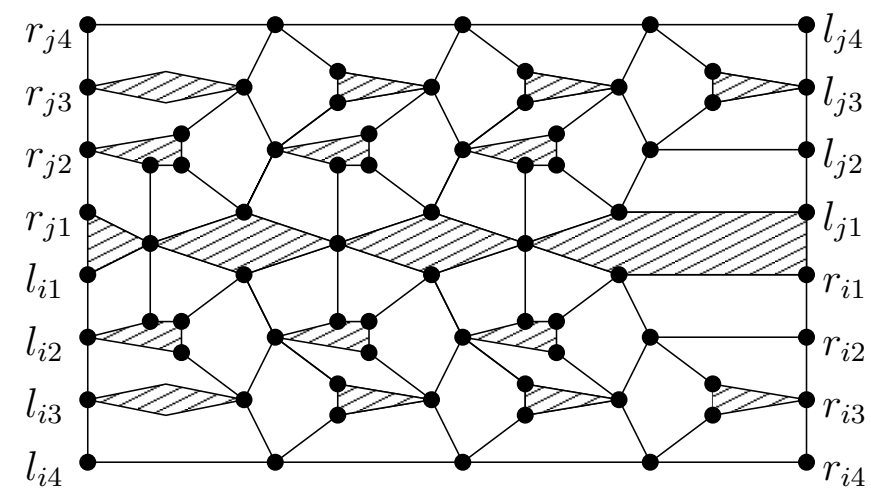

(a) Edge component

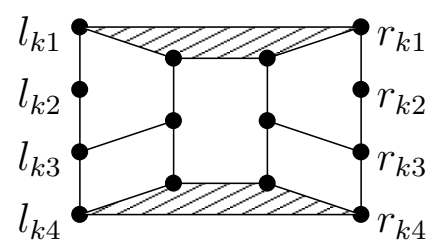

(b) Segment connection

Figure 12: Components for the construction with girth 5

\section{References}

[1] V. Chvátal. Recognizing decomposable graphs. Journal of Graph Theory, 8:51-53, 1984.

[2] R. Diestel. Graph Theory. Number 173 in Graduate Texts in Mathematics. SpringerVerlag, 1997.

[3] M. R. Garey and D. S. Johnson. Computers and Intractability. A Guide to the Theory of $\mathcal{N} \mathcal{P}$-completeness. Freeman, 1979.

[4] M. R. Garey, D. S. Johnson, and L. Stockmeyer. Some simplified $\mathcal{N} \mathcal{P}$-complete graph problems. Theoretical Computer Science, 1:237-267, 1976.

[5] M. Patrignani and M. Pizzonia. The complexity of the matching-cut problem. In Lecture Notes in Computer Science, volume 2204, pages 284-295, 2001. 